\title{
Comparison of ultrasonography-guided pectoral nerve block with patient-controlled analgesia for breast surgery patients
}

\author{
Gökhan Işık Serçe $\odot$, Evrim Kucur Tülübaş®
}

Department of Anesthesiology and Critical Care Medicine, Bakırköy Dr. Sadi Konuk Training and Research Hospital, İstanbul, Turkey

\begin{abstract}
Objectives: Pectoral nerve block is an effective method that can be applied for analgesic purposes in breast surgery. In this prospective study we aimed to compare the postoperative analgesic efficacy of pectoral nerve block for postoperative analgesia and patient-controlled analgesia (PCA) for patients undergoing breast surgery in terms of the incidence of nausea and vomiting.

Methods: The study included 93 patients who underwent ASA I-II anesthesia and breast surgery. Group 1 was PCA group, group 2 was PECS (Pectoral Nerves) block group. PECS block was administered as PECS-1 and PECS-2 block under the guidance of ultrasonography. Patients investigated in the study had postoperative monitoring forms examined for vital signs and visual analog scale (VAS) scores in the $1^{\text {st }}, 6^{\text {th }}$ and $24^{\text {th }}$ hours.

Results: When the pectoral nerve block with bupivacaine under ultrasound guidance was compared with PCA device, there were significant reductions in VAS score at 24 hours.(1.53 vs 4.27, 1.10 vs 3.27 and 0.90 vs 1.93 , respectively; $p=0.0001)$. Although there was no significant difference in terms of vomiting, there was a difference in favor of pectoral nerve block especially at the postoperative 6th hour in terms of nausea.

Conclusions: Pectoral nerve block can be used in the patients undergoing breast surgery due to the lower visual analog score and nausea incidence in the postoperative period.

Keywords: Breast surgery, pectoral nerve block, ultrasonography, patient-controlled analgesia
\end{abstract}

B reast cancer is the most common cancer type among women. In the United States of America, 1 out of every 8 women will be diagnosed with breast cancer [1]. All breast surgeries, led by modified radical mastectomy, are limited by severe acute postoperative pain and painful shoulder movements [2].

Thoracic paravertebral block is the most commonly used block to ensure analgesia among patients operated for breast cancer. However, patients administered thoracic paravertebral block feel pain after surgery in both the axilla and upper extremities. This is because this block does not effectively block the medial and lateral pectoral nerves [3-10]. Pectoral nerve block enters the scenario to solve these problems. Pectoral nerve block administers local anesthetic between the muscles of the anterior wall of the chest; the pectoralis major, pectoralis minor and serratus anterior muscles; and provides very effective analgesia for breast surgery.

In the postoperative period vomiting may cause many unwanted results such as fluid electrolyte loss, aspiration of vomited material in the airway, straining of suture lines and increased intraocular and intraabdominal pressure [11]. All of these results disrupt the patient's comfort and may increase the duration of hospital stay and involve serious costs. After these sur- 
geries the use of high-dose opioids for analgesic purposes increases the incidence of nausea and vomiting. When pectoral nerve block is correctly administered, it reduces opioid requirements to a minimum due to strong analgesic effect and reduces the incidence of nausea-vomiting. In this prospective study we aimed to compare the postoperative analgesic efficacy of pectoral nerve block for postoperative analgesia and patient-controlled analgesia (PCA) for patients undergoing breast surgery in terms of the incidence of nausea and vomiting.

\section{METHODS}

After this study received permission from University of Health Sciences, Bakırköy Dr. Sadi Konuk Traning and Research Hospital Local Ethics Committee numbered 2016-02-06, the records of a total of 93 patients who underwent modified radical mastectomy with general anesthesia at Bakırköy Dr. Sadi Konuk Education and Research Hospital General Surgery Clinic from 01.03.2016 to 01.07.2016 were investigated. After the approval of the ethics committee, the study was applied prospectively. The patients investigated had general 3 anesthesia induction with fentanyl $2 \mathrm{mcg} / \mathrm{kg}$ (according to ideal body weight [IBW]) and propofol $2-3 \mathrm{mg} / \mathrm{kg}$ with muscle relaxant of rocuronium $0.6 \mathrm{mg} / \mathrm{kg}$ (according to true body weight [TBW]). Anesthesia maintenance used 40\% O2+60\% air with $2 \%$ sevoflurane and remifentanil (0.1-0.3 $\mathrm{mcg} / \mathrm{kg} / \mathrm{min}$ ) infusion. All patients were administered $1 \mathrm{~g}$ paracetamol $+100 \mathrm{mg}$ tramadol and $8 \mathrm{mg}$ ondansetron before the start of the surgical procedure.

We included ASA I and II female patients between the ages of 18 and 65 years undergoing modified radical mastectomy for carcinoma breast after obtaining of participants. Patients with local anesthetic allergy, locally advanced breast malignancies with skin ulceration or infiltration of chest wall, patients on anticoagulants, bleeding dyscrasias and anormaly liver function tests were excluded from the study.

Accidental numbers were produced in order to prevent selection bias. Group 1 was PCA group, group 2 was PECS group. Random numbers made MedCalc 18.2.1. Breast surgery team consists of 3 surgical specialists and 1 anesthesiologist. Patients who underwent only radical mastectomy were included in this study.
The pectoral nerve block was performed both as PECS- 1 and PECS- 2 blocks between the $3^{\text {rd }}$ and $4^{\text {th }}$ intercostal spaces, in the midclavicular line, accompanied by ultrasonography. It was performed before incision, after intubation. For PECS-1 block, $10 \mathrm{ml}$ of $0.25 \%$ bupivacaine was administered between the pectoralis major and pectoralis minor muscles. For PECS2 block, in the same plane, $20 \mathrm{ml} 0.25 \%$ bupivacaine was administered between the serratus anterior and pectoralis minor muscles. A LOGIQE GE Healthcare brand (GE Medical Systems, Phoenix - USA) USG device and USG probe (12 MHz, Linear) were used with a Pajunk brand $50 \mathrm{~mm}$ blunt-tipped peripheral nerve block needle (Stimuplex A ${ }^{\circledR}$ B. Braun 82 Melsungen AG, JAPAN).

Patients using a patient-controlled analgesia (PCA) device for postoperative analgesia had the device set up after extubation at the end of the operation with IV. The medication solution used in the PCA device is standard for our clinic and is set with tramadol $300 \mathrm{mg} / 100 \mathrm{ml}$, bolus $5 \mathrm{mg}$, lock time 15 minutes and basal infusion $10 \mathrm{mg} /$ hour. When the VAS score was above $4,0,1 \mathrm{mg} / \mathrm{kg}$ of morphine IV was administered.

Patients investigated in the study had postoperative monitoring forms examined for vital signs [systolic arterial pressure (SAP), diastolic arterial pressure (DAP), mean arterial pressure (MAP)], vomiting presence/absence, nouse presence/absence and visual ana$\log$ scale 4 (VAS) scores in the $1^{\text {st }}, 6^{\text {th }}$ and $24^{\text {th }}$ hours. Clinical nurses were evaluated for nausea and vomiting and the presence / absence of nausea and vomiting was recorded. VAS scores of the patients were evaluated by the anesthesiologist and the checked by the authors. PCA use of the patients was evaluated by the anesthesiologist.

\section{Statistical Analysis}

Statistical analysis of data used the SSPS 23 program. Normal distribution of data was tested with the Kolmogorov-Smirnov test. Mean, standard deviation, median, minimum and maximum values were used for descriptive statistics. Frequency and percentage values were calculated for categorical variables. The Mann Whitney U test was used to compared to groups with non-normal distribution, while the chi-square or Fisher exact probability test was used to compare categorical variables. The Cochran $\mathrm{Q}$ test and the Friedman test for variation over time was used. $P<0.05$ was ac- 
cepted as statistically significant. For the analysis results with $80 \%$ power and moderate effect levels, the necessary sample number for each group was identified as 30, for a total of 60 people (PECS and PCA groups). Power analysis used the PASS 15 (Power Analysis and Sample Size Software, 2017) program.

\section{RESULTS}

The study included 93 patients operated modified radical mastectomy the General Surgery Clinic of Bakırköy Dr. Sadi Konuk Education and Research Hospital. The 93 patients determined by screening were all women. Five patients in ASA III risk group and 5 patients above the age of 65 years were excluded from the study. Our prostective study investigated the pectoral nerve block (PECS) and patient-controlled analgesia (PCA) methods for postoperative analgesia in terms of analgesic efficacy and postoperative nausea-vomiting. Of the 43 patients with pectoral nerve block administered, 10 had insufficient monitoring and 3 patients were transferred to the intensive care unit and were excluded from the study. Of the 40 patients with patient-controlled analgesia device, 6 patients had the device removed due to severe nausea and vomiting in the early postoperative period, and 4 had insufficient postoperative monitoring and were excluded from the study. Finally, patient data of 30 cases in the PECS group and 30 cases in the PCA group for a total of 60 patients were investigated statistically.

The mean age in the PCA group was 48 years, while it was 53 years in the PECS group. The PCA comprised 11 patients in ASA I and 19 patients in ASA II. The PECS group comprised 8 patients in ASA I and 22 patients in ASA II. There was a statistically significant difference identified between the groups in terms of mean SAP in the 1st hour $(p<0.05)$. In the PCA group the systolic blood pressure in the 1 st hour was significantly higher compared to the PECS group. There was no statistically significant difference between the groups for mean SAP in the $6^{\text {th }}$ and $24^{\text {th }}$ hours $(p>0.05)$. There was no statistically significant difference identified between the groups in terms of mean DAP in the $1^{\text {st }}, 6^{\text {th }}$ and $24^{\text {th }}$ hours $(p>0.05)$ (Table 1).

PCA group received $30 \mathrm{mg}$ of the first hour, 110 $\mathrm{mg}$ at 6 hours and $315 \mathrm{mg}$ of tramadol at 24 hours.

There was no significant difference identified between the nausea rates in the block and PCA groups in the $1^{\text {st }}$ hour $(p=1.000)$. The nausea rates all patients were similar in the first hour. In the 6th hour there was no nausea among the block patients. Among 30 patients with PCA, 18 (60\% of patients) had nausea. When examined in terms of nausea in the 6th hour, pectoral nerve block effectively prevented nausea after breast surgery. PCA administration was understood to be deficient in this regard. There was no nausea in both the PECS group and PCA group in the 24th hour. $P$ value could not be calculated as there was no patient with nausea in either group (Table 2). There was no significant difference identified between the PECS and PCA groups in terms of vomiting in the 1 st hour $(p=$ $1.000)$. The vomiting rates in the patient and PCA

Table 1. Assessment of SPB and DBP measurements

\begin{tabular}{lccc}
\hline & $\begin{array}{c}\text { PCA } \\
(\text { Mean } \pm \text { SD) }\end{array}$ & $\begin{array}{c}\text { PECS } \\
(\text { Mean } \pm \text { SD) }\end{array}$ & p value \\
\hline SAP $(\mathbf{m m} / \mathbf{H g})$ & & & \\
\hline 1st hour & $148.67 \pm 19.61$ & $133.33 \pm 19.49$ & $\mathbf{0 . 0 4 8}$ \\
\hline 6th hour & $137.33 \pm 17.60$ & $135.17 \pm 18.31$ & 0.454 \\
\hline 24th hour & $131.00 \pm 16.89$ & $137.17 \pm 17.89$ & 0.410 \\
& DAP $(\mathbf{m m} / \mathbf{H g})$ & & \\
\hline 1st hour & $83.67 \pm 7.06$ & $78.17 \pm 10.79$ & 0.227 \\
6th hour & $77.50 \pm 6.40$ & $79.67 \pm 7.87$ & 0.917 \\
\hline 24th hour & $76.67 \pm 15.72$ & $80.17 \pm 7.20$ & 0.408 \\
\hline
\end{tabular}

*Fisher exact probability test, **Mann Whitney U test, PCA $=$ Patient-Controlled Analgesia, PECS $=$ Pectoral

Nerves block, SBP $=$ Systolic blood pressure, DAP $=$ Diastolic blood pressure 
Table 2. Comparison of nausea, vomiting and VAS scores between groups

\begin{tabular}{lccc}
\hline & $\begin{array}{c}\text { Nausea } \\
\text { 1st hour }\end{array}$ & $\begin{array}{c}\text { Nausea } \\
\text { 6th hour }\end{array}$ & $\begin{array}{c}\text { Nausea } \\
\text { 24th hour }\end{array}$ \\
\hline PCA & 9 & 18 & 0 \\
PECS & 5 & 0 & 0 \\
\hline $\boldsymbol{p}$ value* & 1.00 & $\mathbf{0 . 0 1}$ & - \\
& Vomiting & Vomiting & Vomiting \\
1st hour & 9 & 6th hour & 24th hour \\
PCA & 3 & 0 & 0 \\
PECS & 0.143 & 0 & 0 \\
\hline $\boldsymbol{p}$ value* & VAS & - & - \\
& $\mathbf{1 s t}$ hour & VAS & VAS \\
PCA (mean) & 4.27 & $\mathbf{6 t h ~ h o u r ~}$ & $\mathbf{2 4 t h}$ hour \\
PECS (mean) & 1.53 & 3.27 & 1.93 \\
P** & $\mathbf{0 . 0 0 0 1}$ & 1.10 & 0.90 \\
\hline
\end{tabular}

*Fisher exact probability test, **Mann Whitney U test, PCA = Patient-Controlled Analgesia, PECS = Pectoral Nerves block

groups were similar in the $1^{\text {st }}$ hour. There was no vomiting in the PECS and PCA groups in the $6^{\text {th }}$ and $24^{\text {th }}$ hours. As a result $p$ values could not be calculated. According to analysis to identify differences in the VAS scores for the PECS and PCA groups in the $1^{\text {st }}, 6^{\text {th }}$ and $24^{\text {th }}$ hours, there were significant differences identified in the 1st hour $(p=0.0001), 6^{\text {th }}$ hour $(p=0.0001)$ and $24^{\text {th }}$ hour $(p=0.0001)$. For all measurements the PECS group were observed to have lower VAS scores (Table 2).

\section{DISCUSSION}

In the present study, when the PECS and PCA were compared, the VAS score in the PECS group was statistically significantly lower at the 1st, 6th and 24th hours. In this case, the use of PECS for postoperative analgesia seems to be effective. Another result is that the nausea is significantly higher in the PCA group than the PECS group at the 6th hour. There 6 was no significant difference in term of vomiting in the 1 st, $6^{\text {th }}$ and $24^{\text {th }}$ hours between the groups.

Postoperative pain is an acute pain beginning with surgical trauma, and ideally will gradually reduce to end with wound healing. If postoperative pain is not treated, it may cause serious systemic side effects like atelectasis, hypoxemia and pneumonia in the pulmonary system; tachycardia, mainly, along with cardiac arrhythmia, hypertension, myocardial ischemia and thromboembolic events in the cardiovascular system; gastric stasis, paralytic ileus, nausea and vomiting in the gastrointestinal system; urinary retention in the genitourinary system; increased catabolism and hyperglycemia in the endocrine system; immunosuppression and tendency for infections in the immune system; delayed wound healing and muscle spasms [12-14]. The occurrence of these negative effects of postoperative pain mentioned above have led to approaches to postoperative pain treatment becoming important in anesthesia practice.

However, in spite of the latest developments in analgesia administration methods and production of analgesic mediations with new pharmacokinetic profiles, current evidence shows that postoperative pain is not sufficiently treated [13].

Together with a variety of conservative methods for analgesia management for breast cancer operations, thoracic paravertebral block used to be ensure analgesia. However, as thoracic paravertebral block does not effectively block the medial and lateral pectoral nerves, its use for analgesic aims in breast sur- 
gery is limited. Additionally, thoracic paravertebral block administration includes the risk of serious pneumothorax, spinal cord trauma, sympathetic block and linked hypotension [3-8]. Due to these complications and insufficient analgesic power, paravertebral block administration is avoided. Pectoral nerve block began as an alternative.

Similar to the present study, the study by Bashandy et al. [14], in which PECS block was compared with the PCA group, it was reported that, in the post-anesthesia care unit, nausea and vomiting as well as sedation scores were lower in the PECS group when compared to the control group and the VAS score significantly decreased in the PECS group. In addition, the combined Pecs I and II block is a simple, easy-tolearn technique that produces good analgesia for radical breast surgery. Unfortunately, even if nausea and vomiting decreased in the PECS group also in the present study, not to evaluate them with PONV, which is more specific, remains as a limitation in this study. In another publication, Neeth et al. [17] compared PECS block with general anesthesia and reported that PECS block reduced the need for postoperative analgesia and led to a decrease in the VAS score. However, unlike the present study, they did not find any difference in terms of nausea and vomiting in the postoperative evaluation performed with PONV. Another study that supports the results obtained in this study and reported no difference in nausea and vomiting, but reported a decrease in VAS value, is the study by Morioka et al. [16]. In the present study, however, nausea was significantly higher in the PCA group at 6th hour. Kulhari et al. [17] compared the PECS block with thoracic vertebral block. Although it does not have the same characteristics with the present study, given the evaluation in terms of PONV, the vomit and nausea incidence was found to be low in both groups for block suitability and the decrease in the use of opioids was seen to be directly associated with the low incidence of vomit and nausea.

Contrary to the results obtained in the present study, Cros et al. [18] reported that PECS I was not better than a saline placebo in the presence of multimodal analgesia for breast cancer surgery in the breast surgery group that underwent only in the PEC 1 block. However, in addition to PECS 1 block, PEC 2 block was also implemented in the patient group in the present study. Since the PECS 1 block is insufficient in mastectomies where axillary dissection is implemented alone, the block of thoracicus longus nerve was described as the added PECS 2 block [19, 20].

\section{Limitations}

There are some limitations when evaluating these results. The most important of these is that the results are not prospective. In others, however, we did not measure the total amount of tramadol consumed by patients in PCA, we should have used PONV score for vomiting and nausea. The high systolic arterial pressure in the PCA group at the first hour depended on the VAS score being above 4 . In this case, we could not give enough analgesia within 1 hour. Therefore, we administered the rescue dose.

\section{CONCLUSION}

In conclusion, we believe pectoral nerve block within a multimodal approach especially using USG guidance provides reliable and effective postoperative analgesia for patients undergoing breast surgery.

\section{Authors' Contribution}

Study Conception: GIS, EKT; Study Design: GIS, EKT; Supervision: GIS, EKT; Funding: GIS, EKT; Materials: GIS, EKT; Data Collection and/or Processing: GIS, EKT; Statistical Analysis and/or Data Interpretation: GIS, EKT; Literature Review: GIS, EKT; Manuscript Preparation: GIS, EKT and Critical Review: EKT.

\section{Conflict of interest}

The authors disclosed no conflict of interest during the preparation or publication of this manuscript.

Authors declare that they have no conflict of interest.

\section{Financing}

The authors disclosed that they did not receive any grant during conduction or writing of this study.

\section{REFERENCES}

1. Desroches J, Grabs U, Grabs D. Selective ultrasound guided pectoral nerve targeting in breast augmentation: How to spare the 
brachial plexus cords? Clin Anat 2013;26:49-55.

2. Fecho K, Miller NR, Merrit SA, Klauber-Demore N, Hultman $\mathrm{CS}$, Blau WS. Acute and persistent postoperative pain after breast surgery. Pain Med 2009;10:708-15.

3. Perlas A, Niazi A, McCartney C, Chan V, Xu D, Abbas S. The sensitivity of motor response to nerve stimulation and paresthesia for nerve localization as evaluated by ultrasound. Reg Anesth Pain Med 2006;31:445-50.

4. Ono K, Danura T, Koyama Y, Hidaka H. [Combined use of paravertebral block and general anesthesia for breast cancer surgery]. Masui 2005;54:1273-6. [Article in Japanese]

5. Kairaluoma PM, Bachmann MS, Korpinen AK, Rosenberg PH, Pere P. Single-injection paravertebral block before general anesthesia enhances analgesia after breast cancer surgery with and without associated lymph node biopsy. Anesth Analg 2004;99:1837-43.

6. Moskowitz AS, Goodman RR. Autoradiographic analysis of mu1, mu2, and delta opioid binding in the central nervous system of C57BL/6BY and CXBK (opioid receptor-deficient) mice. Brain Res 1985;360:108-16.

7. Arunakul P, Ruksa A. General anesthesia with thoracic paravertebral block for modified radical mastectomy. J Med Assoc Thai 2010;93.Suppl 7: S149-53.

8. Boughey JC, Goravanchi F, Parris RN, Kee SS, Kowalski AM, Frenzel JC, et al. Prospective randomized trial of paravertebral block for patients undergoing breast cancer surgery. Am J Surg 2009;198:720-5.

9. Moller JF, Nikolajsen L, Rodt SA, Ronning H, Carlsson PS. Thoracic paravertebral block for breast cancer surgery: a randomized double-blind study. Anesth Analg 2007;105:1848-51.

10. Schnabel A, Reichl SU, Kranke P, Pogatzki EM, Zahn PK. Efficacy and safety of paravertebral blocks in breast surgery: a meta-analysis of randomized controlled trials. Br J Anaesth 2010;105:842-52.

11. Dinges HC, Otto S, Stay DK, Bäumlein S, Waldmann S,
Kranke P, et al. Effect rates of opioids in equianalgesic doses via intravenous patient-controlled analgesia: a systematic review and network meta-analysis. Anesth Analg 2019;129:1153-62.

12. Nathanson SD, Wachna DL, Gilman D, Karvelis K, Havstad S, Ferrara J. Pathways of lymphatic drainage from the breast. Ann Surg Oncol 2001;8:837-43.

13. Kenny GNC. Ketorolac trometamol--a new non-opioid analgesic. Br J Anaesth 1990;65:445-7.

14. Bashandy GMN, Dina NA. Pectoral nerves I and II blocks in multimodal analgesia for breast cancer surgery: a randomized clinical trial. Reg Anesth Pain Med 2015;40:68-74.

15. Neethu M, Pandey RK, Sharma A, Darlong V, Punj J, Sinha, et al. Pectoral nerve blocks to improve analgesia after breast cancer surgery: a prospective, randomized and controlled trial. J Clin Anesthesia 2018;45:12-7.

16. Morioka H, Kamiya Y, Yoshida T, Baba H. Pectoral nerve block combined with general anesthesia for breast cancer surgery: a retrospective comparison. JA Clin Rep 2015;1:5.

17. Kulhari S, Bharti N, Bala I, Arora S, Singh G. Efficacy of pectoral nerve block versus thoracic paravertebral block for postoperative analgesia after radical mastectomy: a randomized controlled trial. Br J Anaesth 2016;117:382-6.

18. Cros J, Senges P, Kaprelian S, Desroches J, Gagnon C, Labrunie A, et al. Pectoral I block does not improve postoperative analgesia after breast cancer surgery: a randomized, double-blind, dual-centered controlled trial. Reg Anesh Pain Med 2018;43:596604.

19. Guzzetti L, Danelli G, Ricci B, Torrano V, Russo G, Fusco P, et al. An observational analysis about novel chest wall blocks (PECS and SERRATUS) during breast surgery. Austin J Surg 2016;3:1-5.

20. Eldeen HMS. Ultrasound guided pectoral nerve blockade versus thoracic spinal blockade for conservative breast surgery in cancer breast: a randomized controlled trial. Egypt J Anaesth 2016;32:29-35. 